Of patients treated for volvulus (median follow up 10 months), 5 (55\%) had PEC removal after 3 months without recurrence to date, whilst $2(22 \%)$ had recurrent volvulus following PEC removal requiring further intervention (surgery 1, further PEC 1). One patient required regular venting from the PEC, hence tube not removed.

Two patients with pseudo-obstruction have ongoing venting via their PEC tube, whereas one patient removed their PEC 3 weeks post insertion. Despite forcible removal, there was no observed faecal peritonitis.

Conclusions Sigmoidopexy-assisted PEC appears to be a safe and effective technique, with no significant post-procedural complications in this case series. Two patients developed recurrent sigmoid volvulus after tube removal, suggesting a need to leave tubes in situ for longer than the 3 months used in our initial protocol.

\section{PTH-106 PATIENT PERCEPTION OF FIT IN THE DIAGNOSTIC PATHWAY FOR COLORECTAL CANCER: A MIXED METHOD STUDY}

\begin{abstract}
${ }^{1,2}$ Theo Georgiou Delisle*, ${ }^{1}$ Nigel D'Souza, ${ }^{2}$ Bethan Davies, ${ }^{3}$ Sally Benton, ${ }^{4}$ Michelle Chen, ${ }^{2}$ Helen Ward, ${ }^{1}$ Muti Abulafi. ${ }^{1}$ Croydon University Hospital, London, UK; ${ }^{2}$ Imperial College London, UK; ${ }^{3}$ Royal Surrey County Hospital, Guildford, UK; ${ }^{4}$ RM Partners, The West London Cancer Alliance, UK
\end{abstract}

\subsection{6/gutjnl-2021-BSG.309}

Introduction The faecal immunochemical test (FIT) is a noninvasive, quantitative immunoassay detecting haemoglobin in faeces. FIT is used in bowel cancer screening in the UK for the asymptomatic population. There is mounting evidence of the high diagnostic accuracy of FIT in patients with suspected colorectal cancer (CRC) symptoms. To date, there is no research on usability and perception of FIT in these patients. The aim of the study was to better understand variation in patient perception and acceptability of FIT for patients with suspected CRC symptoms.

Methods A questionnaire was co-developed with patients and included 21 statements covering four themes: FIT feasibility, faecal aversion, patient knowledge and future intentions. Questionnaires were sent to patients with suspected CRC symptoms participating in the NICE FIT study, a multicentre study determining FIT sensitivity for CRC in symptomatic patients. Logistic regression analysis explored differences in patients' test perception by demographic factors. In addition, semi-structed interviews were conducted with patients who had experienced suspected CRC symptoms and used FIT.

Results 1151 questionnaires (31\% response rate) were analysed; $90.1 \%$ of patients found faecal collection straightforward, (95\% CI 88.3\% - 91.8\%), 76.3\% disagreed FIT was unhygienic (95\% CI 73.7\% - 78.6\%), 78.0\% would prefer FIT to colonoscopy (95\% CI 75.6\% - 80.4\%). Preference for FIT over colonoscopy increased with age (OR 1.02; 95\% CI 1.01 - 1.03). Intention to use FIT again was stronger in patients who successfully used FIT than those who did not (OR 11.19; 95\% CI 2.75 - 45.52) and people of white compared to other ethnicities (OR 3.17; 95\% CI 1.31 - 7.68). 15 patient interviews were completed. Patient interviews identified that patients' perception of GP workload could influence test return preferences with patients concerned that returning FIT directly to GPs could add to GP workload. Patients' perception of missing CRC using FIT, and their personal perception of acceptable risk of missed cancer was variable with evidence that patients' personal experience of cancer risk could influence future behaviour in investigation preference.

Conclusions While most patients found FIT practical and hygienic, perception differences were found. Developing strategies to engage patients with more negative FIT perception should be part of symptomatic FIT pathways. FIT recommendation from GPs should trigger a simple patient pathway with rapid secondary care input.

\section{PTH-107 MANDATORY USE OF FAECAL IMMUNOCHEMICAL TEST WILL IMPROVE TRIAGE FOR LOWER GI CANCER REFERRALS}

${ }^{1}$ Karen Russell*, ${ }^{1}$ Peter Coyne, ${ }^{1}$ Steph Needham, ${ }^{2}$ Katie Elliott, ${ }^{1}$ David Nylander. ${ }^{1}$ Newcastle Upon Tyne NHS Foundation Trust, Newcastle Upon Tyne, UK; ${ }^{2}$ Northern Cancer Alliance, Newcastle upon Tyne, UK

\subsection{6/gutjnl-2021-BSG.310}

Prior to the coronavirus pandemic data from our hospital showed a 39\% increase in referrals through lower GI (LGI) cancer pathway (CWT) from 2018 to 2019 with 30\% having 1 st contact in 14 days. ${ }^{1}$

We structure endoscopy lists and outpatient clinics so that some appointment 'slots' are kept exclusively for LGI CWT. After restart of services post COVID 'lockdown' in April 2020 we attempted to send all patients referred via LGI CWT a FIT kit. We wanted to look at local data to assess the following:

a) Proportion of patients referred via LGI CWT with final cancer diagnosis ie the conversion rate (c-rate). ${ }^{2}$

b) Proportion with first hospital contact within 14 days.

c) Impact of triage using FiT $\geq 10$ as standard for urgent investigation in the absence of alarm symptoms e.g. palpable abdo/rectal mass or weight loss on colorectal cancer (CRC) diagnosis - assessed by calculating negative predictive value (NPV) of a FIT $\geq 10$ for CRC in this cohort.

Method We prospectively collected the following from LGI CWT referrals from $1 / 4 / 20$ to 31/12/20: Patient demographics, referral 'symptom', dates patients referred and first hospital contact ('seen'), FIT result and final diagnosis of cancer.

Results 1591 patients were referred in the period. 1404 (88.2\%) had a FIT result.

Monthly referral numbers shown in figure 1 .

The unadjusted (for choice) median time to first seen was 25 days $(0-92)$; only $17 \%$ seen within 14 days

51 cancers diagnosed in this cohort (includes 1 patient with pancreatic and 1 with possible gallbladder cancer) i.e. a c-rate of $3.2 \%$ for all cancer (3.1\% for CRC). All apart from one of the patients with a final diagnosis of CRC referred via CWT had FiT $\geq 10$. So when considering those with a FiT result, c-rate was $7.8 \%$ for patients with $\mathrm{FIT} \geq 10$ and $0.1 \%$ for $\mathrm{FIT}<10$

Table 1 below shows the main presenting symptom with cancer proportion and FiT level

773 patients $(55.1 \%)$ had a FIT $<10$ and 2 of them had final diagnosis of cancer:

- 1 x metastatic pancreas cancer. Presented with abdominal pain and weight loss 